\title{
New Type of White-light LED Lighting for Illumination and Optical Wireless Communication under Obstacles
}

\author{
Su-il Choi* \\ School of Electronics and Computer Engineering, Chonnam National University, \\ 300 Yongbong-dong, Buk-gu, Gwangju 500-757, Korea
}

(Received July 19, 2012 : revised August 20, 2012 : accepted August 24, 2012)

\begin{abstract}
Visible light communications (VLC) use modern solid-state light-emitting diodes (LEDs) to broadcast information. Emerging white-light LEDs allow the combination of lighting and optical wireless communication in one optical source. In this paper, a new LED lighting design using one-chip-type white LEDs is proposed for efficient illumination and optical wireless communications under the existence of several obstacles. Lighting and communication performance are analyzed to show the effectiveness of the proposed LED lighting. Specifically, the signal-to-noise ratio considering intersymbol interference and the bit-error rate of variable pulse position modulation (VPPM) with dimming control are considered.
\end{abstract}
Keywords: Optical wireless, Visible light communication (VLC), White-light LED, Variable pulse position modulation (VPPM)
OCIS codes : (060.0060) Fiber optics and optical communications; (060.4080) Modulation; (060.4510) Optical communications

\section{INTRODUCTION}

Optical wireless communication (OWC) has the potential to be used as a medium for short-range high-speed wireless communications. Thus, optical wireless communication is a promising supplement for existing radio-frequency (RF) communication. When visible light is used for optical wireless communication, the light source can be used for both illumination and communication simultaneously. Compared with conventional lighting methods, white-light LED has lower power consumption, longer lifetime, smaller size, and high power efficiency. Hence, we consider single-chip type white LED, fabricated using a blue LED chip and a phosphor layer, as the cost effective solution for lighting and communication [1,2].

LEDs can be used as a lighting device and a communication device simultaneously. This dual function is based on the fast switching of LEDs and the modulation of the visible-light waves for free-space communications. White-light LEDs offer considerable 3-dB modulation bandwidth [3, 4]. Since VLC can be a solution of the broadband home network, the IEEE Wireless Personal Area Networks working group, IEEE 802.15.7 Task Group 7, developed a standard for VLC [5]. In Europe, the OMEGA project was also done for the development of a user-friendly home access network [6].

Tanaka et al. [7] proposed an optical wireless data transmission system utilizing LED lighting, and designed five typical configurations of LED lights. Using square shaped LED lights, Komine et al. [8] discussed the fundamental analysis including the influence of reflection and intersymbol interference (ISI) for VLC system. Grubor et al. [9] also used square shaped LED lights to compare the system performances for baseband pulse amplitude modulation and discrete multitone modulation. We propose a new type of LED lights that provides robust optical wireless communication under movable obstacles blocking line of sight (LOS) links between the LED lightings and the receiver.

IEEE802.15.7 VLC standard defines a physical layer (PHY) and a media access control layer for short-range optical wireless communication using visible light in optically transparent media. VLC merges lighting and data communications in applications such as area lighting, signboards, streetlights, vehicles, and traffic signals. VLC standard

\footnotetext{
*Corresponding author: sichoi@chonnam.ac.kr

Color versions of one or more of the figures in this paper are available online.
} 
maps the intended applications to three topologies: peer-to-peer, star, and broadcast. The PHY layer supports multiple PHY types and uses on-off keying (OOK), VPPM, and color-shift keying that have multiple light sources and detectors. Since VPPM is a modulation scheme adapted for pulse width based light dimming, we analyze the bit-error rate (BER) performance of VPPM for the proposed LED lighting.

In this paper, a new type of white-light LED lighting is proposed for efficient illumination and optical wireless communication under several obstacles. Three types of LED lighting are analyzed and compared for illumination and communication. Analytical works such as horizontal illuminance, optical wireless channel bandwidth, and signal to noise ratio (SNR) considering ISI are done in order to show the effectiveness of the proposed LED lighting. Especially, the BER of VPPM under dimming control is done. The rest of this paper is organized as follows. Section II shows the properties of white-light LED and compares three types of LED lighting for the provision of proper illuminance over a maximum percentage of the VLC area under several obstacles. Within Section III, we analyze the performance of the communication channel based on the shapes of LED lighting, and show the BER performance of VPPM under dimming control. Final conclusions are covered in Section IV.

\section{WHITE-LIGHT LED LIGHTING}

We consider a model room, which is shown in Figure 1. The size of the model room is $5 \times 5 \times 3 \mathrm{~m}^{3}$ and LED lamps are installed $2.5 \mathrm{~m}$ above the floor $[8,9]$. The data transmitter is located at the center of the ceiling, and electrical cabling is done between the transmitter and LED lightings. Two electrical cabling scenarios are considered: Good cabling scenario represents a cabling that has equal electrical path lengths between the LED lightings, thus it does not cause ISI. Bad cabling scenario represents a cabling with the electrical path difference between the LED lamps large, thus it cause significant reduction of SNR due to ISI. Several obstacles are installed $1.5 \mathrm{~m}$ above the floor to block LOS links between the LED lightings and the receiver; these square shaped obstacles represent standing human bodies or some movable objects blocking LOS links. The height of the desk is $0.85 \mathrm{~m}$. Therefore, we evaluate the system performance at desktop height.

For proper lighting, a certain brightness of the illuminated surface is required, and for a reliable high-speed data transmission, sufficient optical power is needed. Hence, some basic properties regarding LED lights such as luminous flux, transmitted optical power, and horizontal illuminance expressly need to be considered in the LED lighting design.

The luminous flux is the optical power of the source as perceived by the human eye. From radiation spectrum $p(\lambda)[\mathrm{W} / \mathrm{m}]$, one can obtain the luminous flux $\Phi[\mathrm{lm}]$, of the source by

$$
\Phi=K_{m} \int_{380}^{720} p(\lambda) V(\lambda) d \lambda
$$

where $V(\lambda)$ is the standard luminous curve, $K_{m}$ is the maximum visibility, and the maximum visibility is approximately 683 $1 \mathrm{~m} / \mathrm{W}$ at $\lambda=555 \mathrm{~nm}$. The transmitted optical power $P[\mathrm{~W}]$ is given as

$$
P=\int_{\Lambda_{\min }}^{\Lambda_{\max }} p(\lambda) d \lambda
$$

where $\Lambda_{\min }$ and $\Lambda_{\max }$ are determined by the sensitivity curve of the photodiode. White-light LEDs are based on a blue LED chip topped with a phosphor layer. The luminous flux and the transmitted optical power have a conversion factor of $4.2 \mathrm{~mW} / \mathrm{lm}$ for this type of LEDs.

The illuminance is the brightness of the illuminated surface. For an LED with a Lambertian radiation characteristic, the horizontal illuminance is given by

$$
E_{h}=I_{0} \cos ^{m}(\theta) \cos (\phi) / r^{2}
$$

where $I_{0}=I(\theta=0)=(m+1) \Phi /(2 \pi)$ is the maximal luminous intensity, $\theta$ is the angle of irradiance, $\phi$ is the angle of

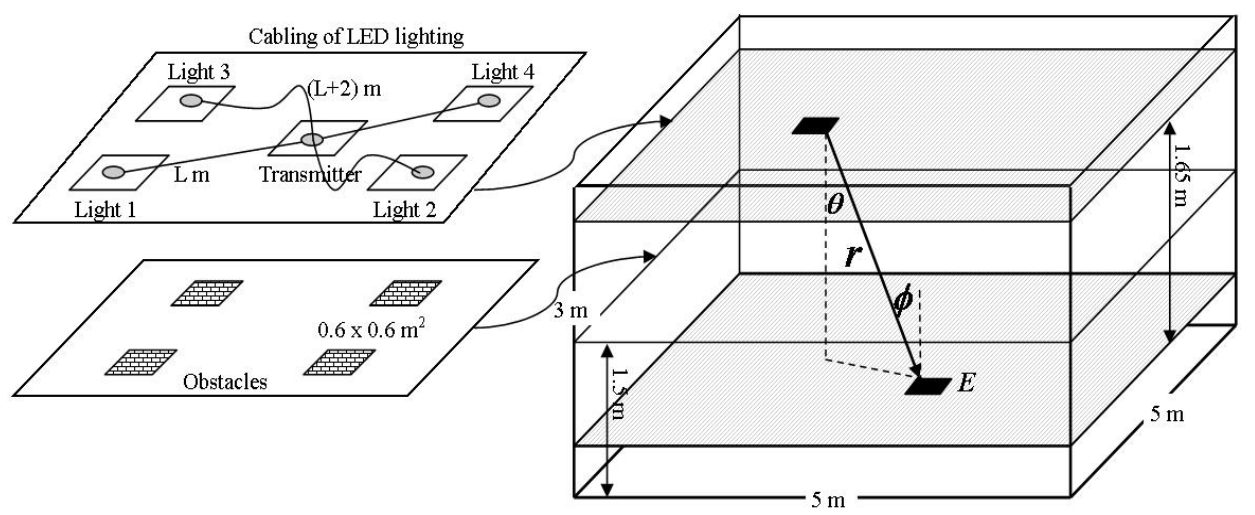

FIG. 1. The model room. 


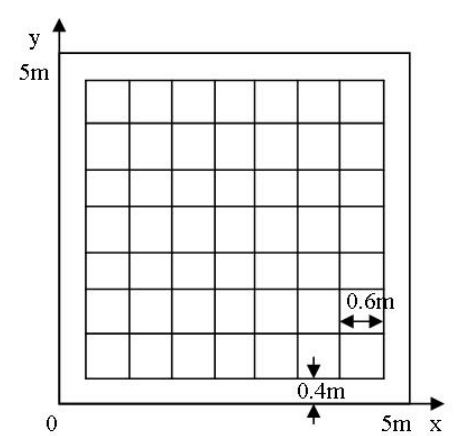

(a)

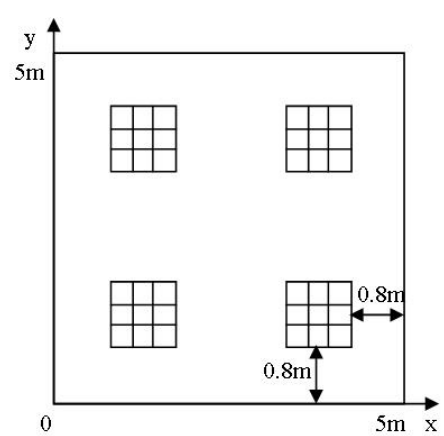

(b)

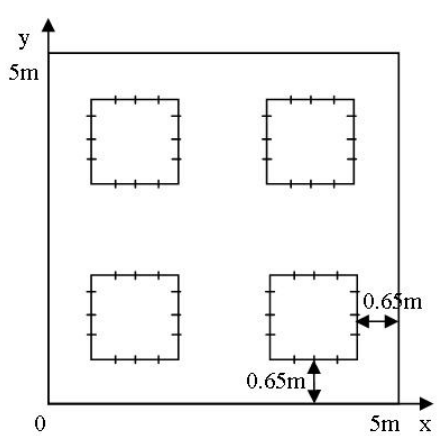

(c)

FIG. 2. Layout of ceiling lighting design: (a) type A, (b) type B, (c) type C.

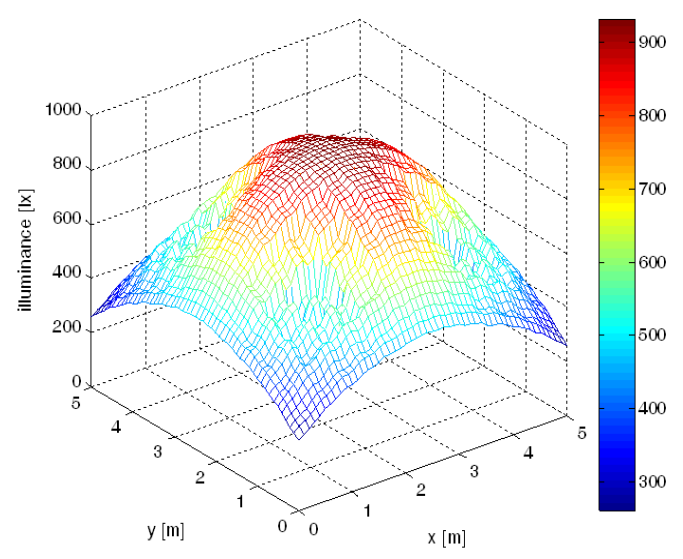

(a)

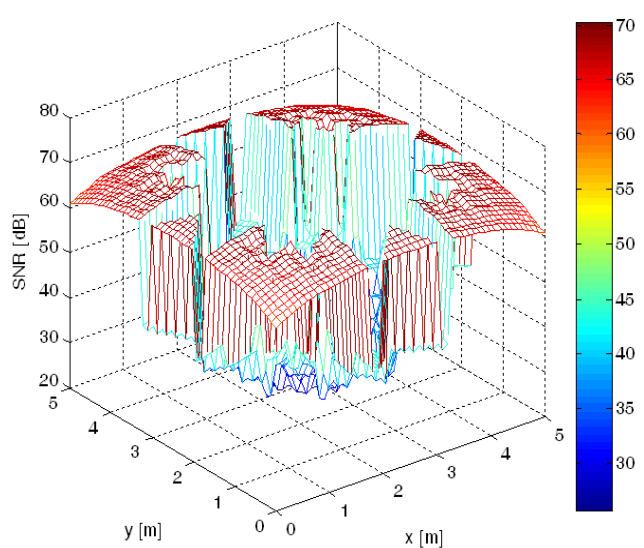

(b)

FIG. 3. Performance distribution for type A at the desktop surface under obstacles: (a) horizontal brightness, (b) SNR considering ISI.

incidence, $r$ is the distance between an LED and a detector's surface, $m=-1 / \log _{2}\left(\cos \theta_{\max }\right)$ is the order of Lambertian emission, and $\theta_{\max }$ is the source radiation semiangle.

\subsection{Design of LED Lighting}

In accordance with EN 12464-1, we regard $400 \mathrm{~lx}$ as the minimal brightness for the height of the desktop in the area where the working place is set, and aim for a 200-800 lx span in the whole room [10]. Thus, the target is to design LED lighting in such a way that an illuminance level of $400 \mathrm{~lx}$ is maintained over a maximum percentage of the VLC area.

Three types of LED lighting are considered for analysis of the VLC channel and the ceiling layout of LED lighting is shown in Figure 2. Type A has a uniform distribution of LEDs, and type B has square shaped LED lightings [8, 9]. Type $C$ is the designed LED lighting, which has another square shape, to reduce the effect of obstacles for the LOS link; LED chips are placed at the boundaries of the square shaped LED lamps. We consider 64 OSTAR white LED chips (LE CW E3B) for three types of LED lighting, and some simulation parameters are listed in Table 1.

For type A, the distribution of horizontal brightness $E_{h}$ and SNR considering ISI are shown in Figure 3. These distri-
TABLE 1. Simulation parameters

\begin{tabular}{|c|c|c|c|}
\hline Parameters & Type A & Type B & Type C \\
\hline $\begin{array}{l}\text { Maximum } \\
\text { luminous } \\
\text { intensity, } I_{0}\end{array}$ & \multicolumn{3}{|c|}{$140 \mathrm{~cd}$} \\
\hline $\begin{array}{c}\text { Transmitted } \\
\text { optical power, } \\
P_{\text {CHIP }}\end{array}$ & \multicolumn{3}{|c|}{$978 \mathrm{~mW}$} \\
\hline $\begin{array}{l}\text { Semi-angle at } \\
\text { half power, } \\
2 \theta_{\max }\end{array}$ & \multicolumn{3}{|c|}{$130^{\circ}$} \\
\hline $\begin{array}{c}\text { LED } \\
\text { configuration at } \\
\text { the ceiling }\end{array}$ & $\begin{array}{l}\text { Uniformly } \\
\text { distributed }\end{array}$ & $\begin{array}{c}4 \text { square } \\
\text { lamps of } \\
0.9 \times 0.9 \mathrm{~m}^{2}\end{array}$ & $\begin{array}{c}\text { Another } 4 \\
\text { square lamps } \\
\text { of } 1.2 \times 1.2 \mathrm{~m}^{2}\end{array}$ \\
\hline $\begin{array}{l}\text { LED chip } \\
\text { spacing }\end{array}$ & $0.6 \mathrm{~m}$ & $0.3 \mathrm{~m}$ & $0.3 \mathrm{~m}$ \\
\hline $\begin{array}{c}\text { Number of LED } \\
\text { chips }\end{array}$ & \multicolumn{3}{|c|}{64} \\
\hline Obstacle size & \multicolumn{3}{|c|}{$0.6 \times 0.6 \mathrm{~m}^{2}$} \\
\hline
\end{tabular}

butions are obtained at the desktop surface under several 




(a)

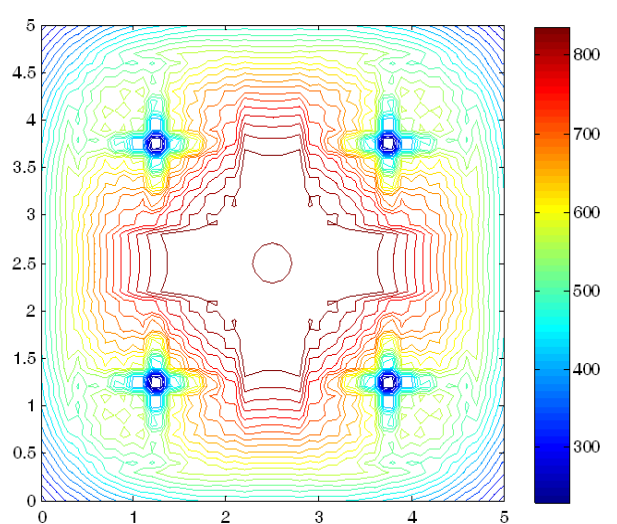

(b)

FIG. 4. Contour distribution of horizontal brightness $E_{h}$ at the desktop surface under obstacles: (a) type B, (b) type C.

obstacles. Type A has a good distribution which covers $92.62 \%$ of the desktop surface for $400 \mathrm{~lx}$, however this cannot be used for optical wireless communication because SNR distribution considering ISI is severely deteriorated even though good cabling is done.

In the presence of obstacles, Figure 4 shows the contour distribution of the horizontal illuminance $E_{h}$ at the desktop surface for type B and type C. In both types of LED lighting, the desired brightness span is met, with a major portion of the plane receiving at least $400 \mathrm{~lx}$. Type B shows the average illuminance as $609.8 \mathrm{~lx}$ and covers $89.85 \%$ of the desktop surface for $400 \mathrm{~lx}$. The horizontal illuminance of type B decays very rapidly below the obstacles, and the minimal illuminance of type $\mathrm{B}$ is $178.5 \mathrm{~lx}$, which causes performance deterioration of the communication channel. In case of type $\mathrm{C}$, the average illuminance is $615.3 \mathrm{~lx}$ and $93.54 \%$ of the desktop surface is covered in regards to $400 \mathrm{~lx}$. The LED lighting for type $\mathrm{C}$ shows superior illuminance distribution compared to that of type B.

\section{ANALYSIS OF COMMUNICATION CHANNEL}

The optical wireless channel model is expressed as follows

$$
y(t)=\gamma x(t) \otimes h(t)+n(t)
$$

where $y(t)$ represents the received signal current, $\gamma$ is the detector responsivity, $x(t)$ represents the transmitted optical pulse, $h(t)$ is the impulse response, $n(t)$ represents the additive white Gaussian noise (AWGN), and the symbol $\otimes$ denotes convolution.

The illuminance at any point of the receiving surface includes LOS as well as reflective lights by walls. The channel response of directed light can be modeled by Dirac pulses whereas the diffused portion can be represented by an integrating-sphere model [11]. The channel frequency response can be written as

$$
H(\omega)=\sum_{i=1}^{N} \eta_{L O S, i} e^{-j \omega \Delta \tau_{L O S j}}+\frac{\eta_{D I F F}}{1+j \omega / \omega_{0}} e^{-j \omega \Delta \tau_{D I F F}}
$$

where $\eta_{L O S}$ and $\eta_{D I F F}$ are the channel gains for the LOS and diffuse signal, respectively; $\Delta \tau_{L O S}$ and $\Delta \tau_{D I F F}$ are the corresponding signal delays, and $f_{0}$ is the cut-off $(3-\mathrm{dB})$ frequency of the purely diffuse channel, and is roughly 10 $\mathrm{MHz}$ for a medium-sized room [11]. The LOS gain from the $\mathrm{i}$-th LED chip is given by

$$
\eta_{L O S, i}=\frac{(m+1) A_{R}}{2 \pi r_{i}^{2}} \cos ^{m}\left(\theta_{i}\right) \cos \left(\phi_{i}\right) \operatorname{rect}\left(\phi_{i} / \phi_{\max }\right)
$$

where $A_{R}$ is the effective receiver surface, $\phi_{\max }$ is the width of the field of vision at a receiver, and $\operatorname{rect}(x)$ is defined as one for $0<x \leq 1$ and zero otherwise. We also assume a Si PIN photodiode with $1 \mathrm{~cm}^{2}$ surface and a concentrator with gain 3 together with a blue spectral filter without passband attenuation, summing up to an effective surface of $A_{R}=3 \mathrm{~cm}^{2}$ [9]. The diffuse signal gain is constant everywhere in the room, and depends only on $A_{R}$ and the room properties as

$$
\eta_{\text {DIFF }}=\frac{A_{R}}{A_{\text {room }}} \frac{\rho}{1-\rho}
$$

where $A_{\text {room }}$ is the room area and $\rho$ is the average reflectivity [11]. The diffuse component has no significant influence on the overall channel bandwidth.

Figure 5 shows the distribution of 3-dB frequencies under obstacles for both types of LED lighting. In both types of LED lighting, spans of available channel bandwidth are well above the maximum modulation bandwidth of 20 MHz. Type B shows the minimum 3-dB cut-off frequency as $65.4 \mathrm{MHz}$ and Type $\mathrm{C}$ has the minimum bandwidth as 


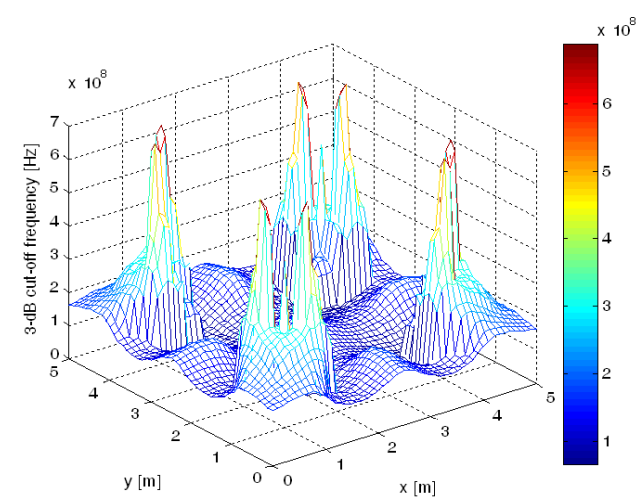

(a)

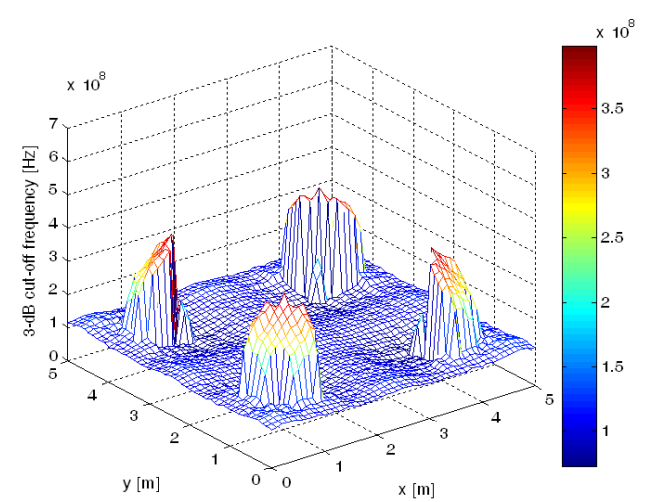

(b)

FIG. 5. 3-dB cut-off frequency (bandwidth) distribution under obstacles: (a) type B, (b) type C.

73.2 MHz. Thus, the channel can be considered flat over the bandwidth of interest.

\subsection{SNR Performance and Intersymbol Interference}

ISI stems from multipath propagation of the emitted signals, and limits the transmission speed. We assume that for each submitted symbol all signals arriving at the receiver with a delay of more than half of the symbol period $T_{\text {sym }}$ after the first signal contribute to ISI [9]. Therefore, the received optical signal power $P_{R, \text { sig }}$ and the received optical noise power due to ISI $P_{R, I S I}$ are given as

$$
\begin{aligned}
& P_{R, s i g}=\sum_{i=1}^{N} P_{R}\left(t_{i} \leq T_{\text {sym }} / 2\right), \\
& P_{R, I S I}=\sum_{i=1}^{N} P_{R}\left(t_{i}>T_{\text {sym }} / 2\right)
\end{aligned}
$$

A SNR expresses the quality of communication. Since the SNR is affected by ISI, the SNR is defined as

$$
S N R_{0}=\gamma^{2} P_{R, s i g}^{2} /\left(N_{0} B+\gamma^{2} P_{R, I S I}^{2}\right)
$$

where $N_{0}$ is the double-sided power spectral density of noise $\left[\mathrm{A}^{2} / \mathrm{Hz}\right]$, the bandwidth $B$ is $20 \mathrm{MHz}$, and the responsivity $\gamma$ of Si-based photodiodes is $\sim 0.28 \mathrm{~A} / \mathrm{W}$ in the blue region. The shot noise stemming from ambient light in regards to windows is assumed to be dominant noise contribution. After blue-light filtering, the noise power spectral density is $N_{0} \approx 10^{-22} \mathrm{~A}^{2} / \mathrm{Hz}$ [9]. The difference of electrical path lengths and/or optical path lengths cause significant reduction of $S N R_{0}$ and limit the transmission rate.

In the case of a good cabling scenario, electrical path lengths between LED lights are equal, Figure 6 shows the $S N R_{0}$ distribution at the desktop surface for type $\mathrm{B}$ and type $\mathrm{C}$. The distribution above $66.6 \mathrm{~dB}$, which means 400 lx, for type B covers $81.5 \%$ of the working plane. The distribution for type $\mathrm{C}$ covers $87.2 \%$ of the working plane, which has an $S N R_{0}$ above $66.6 \mathrm{~dB}$.

In the case of a bad cabling scenario, two LED lightings have an additional $2 \mathrm{~m}$ of cabling to cause ISI, Figure 7 shows the $S N R_{0}$ distribution at the desktop surface for type $\mathrm{B}$ and type $\mathrm{C}$. The electrical path difference between LED lightings creates an increase of the ISI power. Hence, the $S N R_{0}$ distribution for the bad cabling scenario shows severe deterioration of communication performance. The distribution above $15.6 \mathrm{~dB}$, which means $10^{-9} \mathrm{BER}$, for type B covers $92.7 \%$ of the working plane. The distribution for type $\mathrm{C}$ covers $94 \%$ of the working plane, which has an $S N R_{0}$ above $15.6 \mathrm{~dB}$. Figure 6 and Figure 7 show that not only optical path difference but also electrical cabling is an important factor for the design of LED lighting.

\subsection{Modulation Schemes with Dimming Control}

In OOK modulation, the transmitter emits a rectangular pulse of duration $1 / R_{b}$ and of intensity $P$ to signify a one bit, and no pulse to signify a zero bit. The bit-error rate is $B E R=Q\left(\sqrt{S N R_{0}}\right)$ [12]. Then, the power required by OOK to achieve a given BER is given as

$$
P_{\text {OOK }}=\sqrt{N_{0} B+\gamma^{2} P_{R, I S I}^{2}} \cdot Q^{-1}(B E R)
$$

In a pulse-position modulation (PPM) scheme, each symbol interval of duration $T=\log _{2} L / R_{b}$ is partitioned into $L$ sub-intervals, or chips, each of duration $T / L$, and the transmitter sends an optical pulse during one and only one of these chips. Thus, the average power requirement is approximately:

$$
P_{P P M}=\sqrt{\frac{2}{L \log _{2} L}} P_{O O K}
$$

From (11) we see that, 2-PPM has the same sensitivity as OOK, and the required bandwidth to achieve a bit rate $R_{b}$ is approximately $B=2 R_{b}$. VPPM is a modulation scheme adapted for pulse width based light dimming and offers 


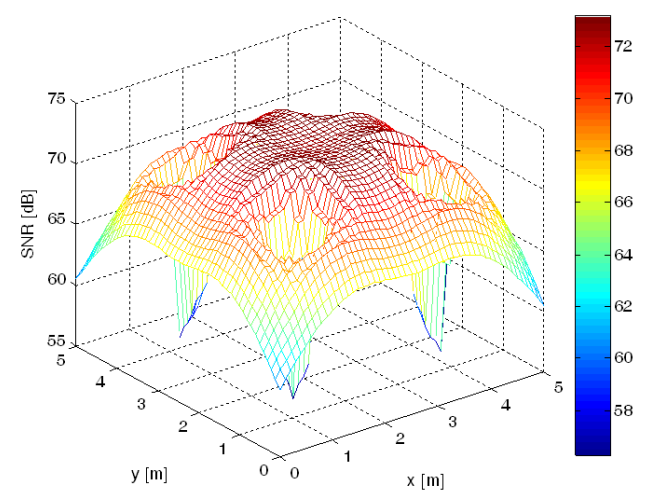

(a)

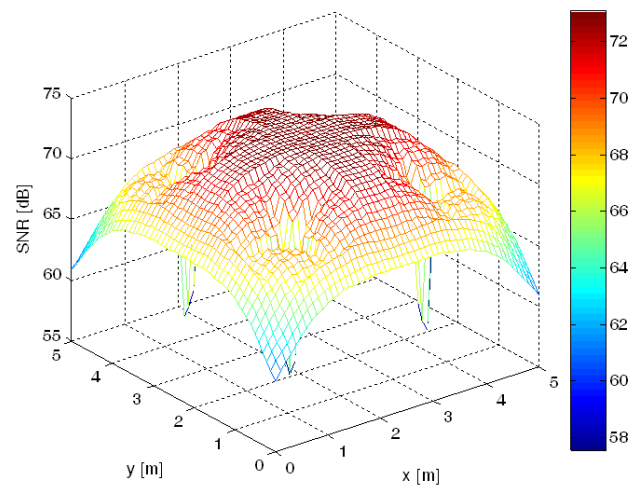

(b)

FIG. 6. Distribution of the SNR on the working plane under obstacles and good cabling: (a) type B, (b) type C.

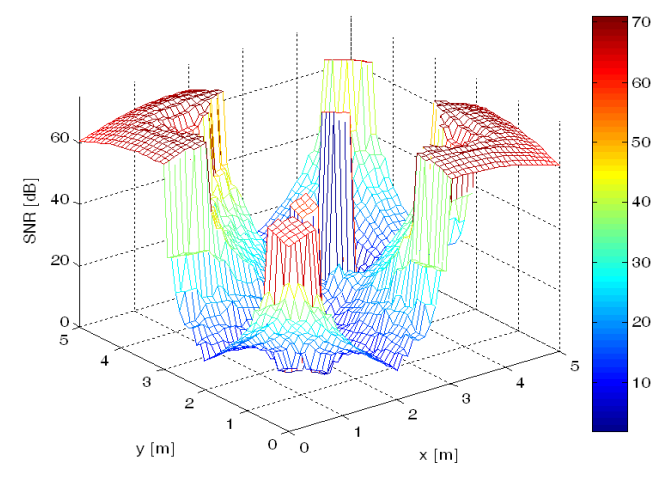

(a)

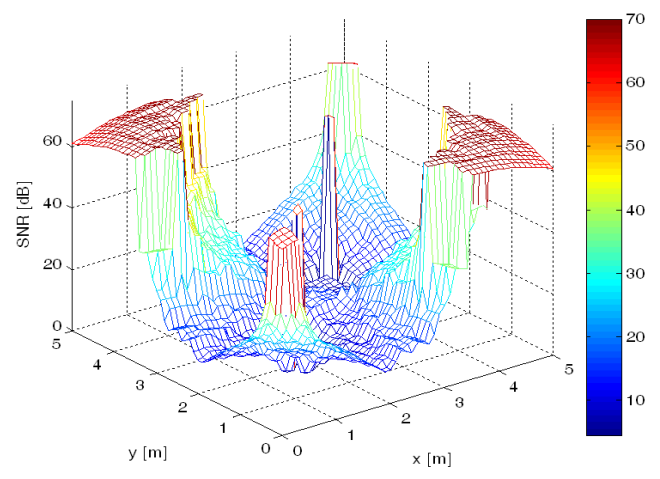

(b)

FIG. 7. Distribution of the SNR on the working plane under obstacles and bad cabling: (a) type B, (b) type C.

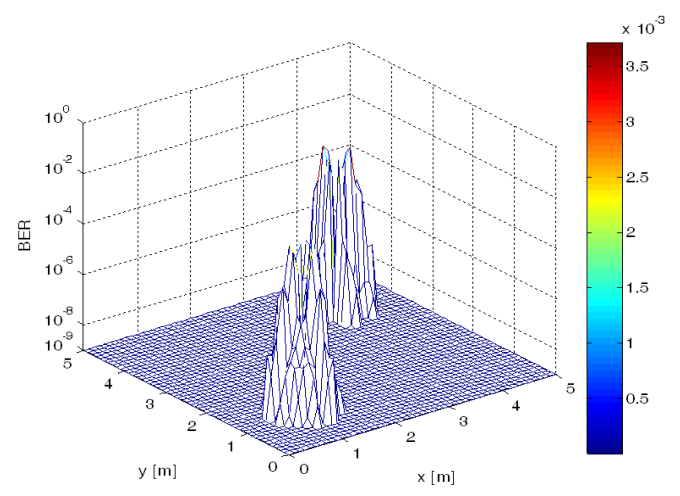

(a)

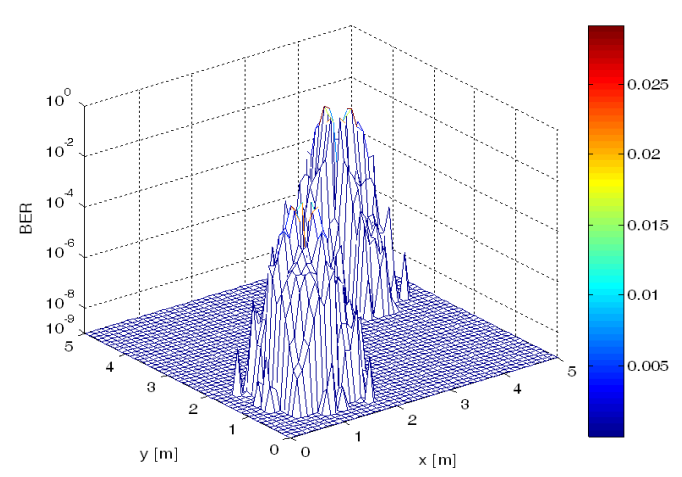

(b)

FIG. 8. The BER distribution of VPPM for type $\mathrm{C}$ on the working plane under obstacles and bad cabling: (a) $\delta=0.5$, (b) $\delta=0.25$ or 0.75 .

protection from intra-frame flicker. VPPM makes use of the characteristics of 2-PPM for non-flicker and pulse width modulation (PWM) regarding dimming control and full brightness. VPPM provides brightness control by varying the duty cycle $\delta$ for $0.1 \leq \delta \leq 0.9$. Therefore, the average power requirement is approximately:

$$
P_{V P P M}=\left\{\begin{array}{cc}
P_{O O K} / \sqrt{2 \delta} & 0.1 \leq \delta \leq 0.5 \\
P_{\text {OOK }} / \sqrt{2(1-\delta)} & 0.5 \leq \delta \leq 0.9
\end{array}\right.
$$

In the case of the good cabling scenario, the BER distribution of VPPM for type $\mathrm{C}$ is below $10^{-9}$. Hence, the distribution of BER performance of VPPM for type C with regards to the bad cabling scenario is considered. Figure 8 shows the BER distribution of VPPM for $R_{b}=10$ Mbps at the desktop surface in the case of the bad cabling scenario and obstacles are applied. When duty cycle is $\delta$ $=0.5,5.99 \%$ of the working plane does not meet the $\mathrm{BER}$ requirement $\mathrm{BER}=10^{-9}$. When the duty cycle is 
changed as $\delta=0.25$ or 0.75 for dimming control, $15.99 \%$ of the working plane does not meet the BER requirement. This means that careful dimming control is required for proper data communication.

\section{CONCLUSION}

In this paper, a new type of white-light LED lighting is proposed for illumination and data communication. Analytical works such as horizontal brightness, 3-db cut-off frequency, and SNR considering ISI on the working plane under obstacles show that the designed LED lighting shows superior performance compared to the existing rectangular LED lighting. It also demonstrates that electrical cabling is an important factor for the design of LED lighting. Moreover, the BER performance of VPPM under obstacles shows that careful dimming control is required for proper data communication.

\section{ACKNOWLEDGMENT}

This work was supported by the National Research Foundation of Korea Grant funded by the Korean Government (NRF-2010-013-D00047).

\section{REFERENCES}

1. T. Komine, Y. Tanaka, S. Haruyama, and M. Nakagawa, "Basic study on visible-light communication using light emitting diode illumination," Proc. ISMOT 2001, 45-48 (Montreal, Canada, Jun. 2001).

2. T. Komine and M. Nakagawa, "Integrated system of white LED visible-light communication and power-line communication," IEEE Trans. on Consumer Electronics 49, 71-79 (2003).

3. J.-H. Kim, C. G. Lee, C. S. Park, S. Hann, and D.-H. Kim, "Visible light communication at $20 \mathrm{Mbit} / \mathrm{s}$ using illumination LEDs," Proc. SPIE 6353, 1-8 (2006).

4. J. Grubor, S. C. J. Lee, K.-D. Langer, T. Koonen, and J. W. Walewski, "Wireless high-speed data transmission with phosphorescent white-light LEDs," Proc. ECOC PD3.6, 1-2 (2007).

5. IEEE Standard P802.15.7, Short-range Wireless Optical Communication Using Visible Light (2011).

6. ICT-213311 OMEGA D4.2b, Physical Layer Design And Specification (2011).

7. Y. Tanaka, T. Komine, S. Haruyama, and M. Nakagawa, "Indoor visible light transmission system utilizing white LED lights," IEICE Trans. on Communications E86-B, 2440-2454 (2003).

8. T. Komine and M. Nakagawa, "Fundamental analysis for visible-light communication system using LED lightings," IEEE Trans. Consum. Electron. 50, 100-107 (2004).

9. J. Grubor, S. Randel, K.-D. Langer, and J. W. Walewski, "Broadband information broadcasting using LED-based interior lighting," J. Lightwave Technol. 26, 3883-3892 (2008).

10. European Standard EN 12464-1, Lighting of Indoor Work Places (2003).

11. V. Jungnickel, V. Pohl, S. Noenning, and C. von Helmolt, "A physical model for the wireless infrared communication channel," IEEE J. Sel. Areas Comm. 20, 631-640 (2002).

12. H. Park and J. R. Barry, "Modulation analysis for wireless infrared communications," in Proc. IEEE Int. Conf. Communications (Seattle, WA, USA, Jun. 1995), pp. 1182-1186. 